\title{
Cosmic Rays from Multifrequency Observations of the Interstellar Emission
}

\author{
Elena Orlando* \\ Hansen Experimental Physics Laboratory and Kavli Institute for Particle Astrophysics and \\ Cosmology, Stanford University, Stanford, CA 94305, USA \\ E-mail: eorlandoestanford.edu
}

\begin{abstract}
Cosmic Rays propagate in the Milky Way and interact with the interstellar medium and magnetic fields. These interactions produce emission that spans the electromagnetic spectrum and is a crucial tool for understanding intensities and spectra of cosmic rays in different regions. Hence observations of this interstellar emission complement information from cosmic ray measurements. We present updates on the study of cosmic ray properties by combining multifrequency observations of the local interstellar emission with latest accurate cosmic ray direct measurements and propagation models. We also make predictions for e-ASTROGAM and AMEGO, proposed gamma-ray missions with a special focus at the $\mathrm{MeV}$ energy range.
\end{abstract}

7th Fermi Symposium 2017

15-20 October 2017

Garmisch-Partenkirchen, Germany

\footnotetext{
${ }^{*}$ Speaker.
} 